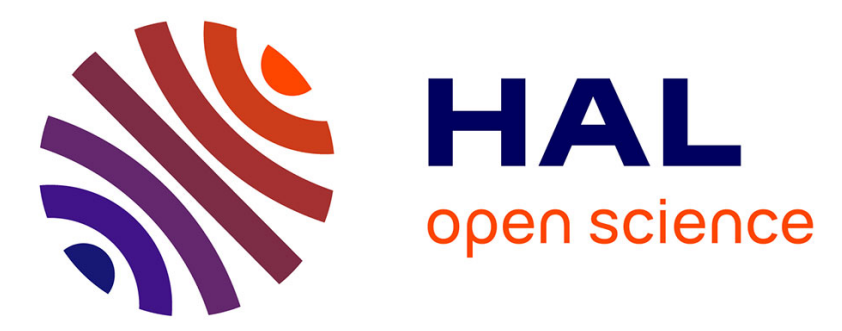

\title{
Wavelength dependence of the orientation of optic axes in $\mathrm{KGW}$
}

Romain Cattoor, Inka Manek-Hönninger, Marc Tondusson, Philippe Veber, Todor K. Kalkandjiev, Daniel Rytz, Lionel Canioni, Marc Eichhorn

\section{- To cite this version:}

Romain Cattoor, Inka Manek-Hönninger, Marc Tondusson, Philippe Veber, Todor K. Kalkandjiev, et al. Wavelength dependence of the orientation of optic axes in KGW. Applied Physics B - Laser and Optics, 2014, 116 (4), pp.831-836. 10.1007/s00340-014-5769-2 . hal-00982635

\section{HAL Id: hal-00982635 https://hal.science/hal-00982635}

Submitted on 23 Feb 2016

HAL is a multi-disciplinary open access archive for the deposit and dissemination of scientific research documents, whether they are published or not. The documents may come from teaching and research institutions in France or abroad, or from public or private research centers.
L'archive ouverte pluridisciplinaire HAL, est destinée au dépôt et à la diffusion de documents scientifiques de niveau recherche, publiés ou non, émanant des établissements d'enseignement et de recherche français ou étrangers, des laboratoires publics ou privés.

\section{다(1)(2)}

Distributed under a Creative Commons Attribution - ShareAlike| 4.0 International 


\title{
Wavelength dependence of the orientation of optic axes in KGW
}

\author{
Romain Cattoor - Inka Manek-Hönninger - Marc Tondusson • \\ Philippe Veber • Todor K. Kalkandjiev • Daniel Rytz • \\ Lionel Canioni · Marc Eichhorn
}

\begin{abstract}
We report on the wavelength dependence of the orientation of the optic axes in monoclinic crystals using undoped and neodymium-doped $\mathrm{KGd}\left(\mathrm{WO}_{4}\right)_{2}$ (KGW) samples. Measurements have been performed over a wide wavelength range from 420 up to $1,580 \mathrm{~nm}$ with more than 70 measurement points. This provides the widest measurement range of the optic axis variation ever reported in literature for $\mathrm{KGW}$ crystals. With such a measurement, which can be performed in principle in all biaxial crystals, the refractive indices values can be checked with a precision down to $10^{-3}$.
\end{abstract}

\section{Introduction}

An old effect commonly called internal conical refraction (ICR) is attracting more and more interest in the last few

R. Cattoor $(\bowtie) \cdot$ M. Eichhorn

Institut Franco Allemand de recherches de Saint Louis (ISL),

5 rue du Général Cassagnou, 68300 Saint Louis, France

e mail: romain.cattoor@isl.eu

R. Cattoor · I. Manek Hönninger · M. Tondusson - L. Canioni Laboratoire Ondes et Matière d'Aquitaine (LOMA), UMR5798 UB CNRS, 351 Cours de la Libération,

33405 Talence, France

P. Veber

Institut de Chimie de la Matière Condensée de Bordeaux (ICMCB CNRS UPR9048), 87 Avenue du Docteur Schweitzer, 33608 Pessac, France

T. K. Kalkandjiev

Conerefringent Optics SL, Av Cubelles 28,

08800 Vilanova i la Geltru, Barcelona, Spain

D. Rytz

FEE GmbH, Struthstr. 2, 55743 Idar Oberstein, Germany years. It has been first predicted by Hamilton in 1832 and quickly observed by Lloyd [1] few months later. Several additional contributions improved the knowledge of this effect, with the observation of the secondary ring [2] and axial spike [3]. Since its prediction by Hamilton, several papers have improved and refined the theory of ICR [4 10]. Furthermore, the authors [11] have analyzed the role of ICR on second harmonic generation in biaxial crystals. The theoretical aspect has been recently revisited by Berry in several papers $[12,13]$. From the theory, one can predict the beam evolution in biaxial crystals. Even the theoretical description of cascaded ICR is reported [14]. ICR appears when a light beam propagates along one of the optic axes. Considering potential applications of this phenomenon, the formation of the characteristic ring by ICR gives access to beam shaping [15 18] and offers new possibilities for innovative configurations in molecule trapping or for some micromachining applications. Even the use of this effect in laser gain media has been explored [19 21]. From those last articles, it results that the orientation of the crystal is important and should be controlled with an accuracy of a few mrad. At present, the most studied material for ICR is $\mathrm{KGd}\left(\mathrm{WO}_{4}\right)_{2}(\mathrm{KGW})$. This choice can be explained by the available good crystal quality of this host matrix and its strong anisotropy compared with other biaxial crystals that are accessible with sufficient quality. The variation of the optic axis orientation in space for different wavelengths can be easily calculated from the refractive indices. However, the precision of the three refractive indices must be very accurate to predict those angles. Unfortunately, it is not easy to determine the three principal refractive indices of biaxial crystals. Several studies have been performed on different biaxial crystals in order to characterize the wavelength dependence of their optical properties. However, the low number of measurement points reduces the 
precision and thus limits the prediction of the results [11, 22 24]. One publication provides impressive results on the wavelength dependence of refractive indices for the monoclinic crystal BIBO [25]. Their measurements of refractive indices were used to calculate the variation of the optic axes with accuracy. However, to the best of our knowledge, no experiments have been performed so far in order to confirm this evolution. In the present paper, we experimentally investigate the wavelength dependence of the optic axes for Nd-doped and undoped KGW within a large wavelength range from 420 to $1,580 \mathrm{~nm}$, and we compare our results with the ones calculated from refractive indices reported in literature [26]. We suggest a small modification of the values of the indices reported in the literature to obtain an satisfactory agreement between calculated and experimentally determined optic axis orientations.

\section{Optic axes}

In biaxial crystals, there are two optic wave axes, located in the $X Z$ plane of the indices surface. The directions $X$, $Y$ and $Z$ represent the axes of the indicatrix having three principal refractive indices $n_{x}, n_{y}$ and $n_{z}$, respectively, where $n_{x}<n_{y}<n_{z}$.

This optical coordinate system is orthogonal and does not coincide with the crystallographic axes. However, in monoclinic KGW crystals, the direction $X$ corresponds to the crystallographic axis $\mathbf{b}$ and the $Z Y$ and a c planes are the same. To set the direction of this optical coordinate system, the angle $\phi$, as shown in Fig. 1, must be known and may depend on temperature and wavelength.

Using X-ray diffraction, Laue patterns of the crystals have been recorded with a CCD camera from Photonic Science for a crystal orientation perpendicular to the entrance facet. The samples were aligned parallel on one plate together with a crystal of silicon. By using the silicon Laue pattern as calibration reference, the plate was perpendicularly aligned to the X-ray beam coming from the

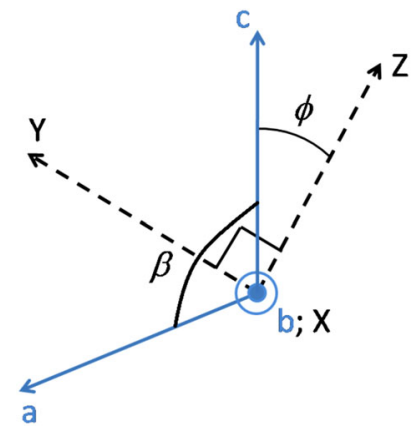

Fig. 1 Orientation of the crystallo optical coordinate with respect to crystallographic axes molybdenum anti-cathode. Using analyzing software based on the Laue method (Orient Express), we could determine all angles between crystallographic axes with respect to our crystal orientation. The mechanical alignment of our samples, relative to the silicon crystal, is the main source of uncertainty. For this purpose, the unit cell parameters of Pujol et al. [26], which are $\mathbf{a}=10.652 \AA, \mathbf{b}=10.374 \AA$, $\mathbf{c}=7.582 \AA$ and $\beta=130.80^{\circ}$, were used. Knowing that for those monoclinic crystals the principal axis of $n_{p}$ $(X)$ corresponds to the crystallographic axis $\mathbf{b}$, we found that the angle $\phi$ between the direction of $n_{z}(Z)$ and the crystallographic axis $\mathbf{c}$ was about $20.0^{\circ} \pm 1$ and $20.7^{\circ} \pm 1$ for undoped $\mathrm{KGW}$ and $\mathrm{Nd}$-doped $\mathrm{KGW}$, respectively. Furthermore with those, records references of angles $V$ at $633 \mathrm{~nm}$ were found to be $43.3^{\circ}$ and $42.1^{\circ}$, respectively. Note that in case of the undoped crystal, a correction has been added in order to take into account the misalignment of this sample at $633 \mathrm{~nm}$ (see next section). The main uncertainty of this method (which is also the main source of uncertainties in our measurements) results in the positioning of the crystal on the translation stage which needs to be perpendicular to X-rays. All those angles calculation is simplified due to monoclinic symmetry. In case of triclinic crystals that are also biaxial, it will be more difficult to determine them.

The angle $V$, between the $n_{z}(Z)$ axis and the optic axis in the $X Z$ plane, can be easily calculated:

$\tan ^{2}(V)=\frac{n_{z}^{2}\left(n_{y}^{2}-n_{x}^{2}\right)}{n_{x}^{2}\left(n_{z}^{2}-n_{y}^{2}\right)}$

Equation 1 is given without any approximation.

Note that in this paper, we discuss only about the optic wave axes (sometimes called binormal) from the index surface (called wave surface in [27]), along which one the ICR takes place. It exists another optic ray axes (also called biradial) from the ray surface. Similar angle $V_{r}$ can be calculated replacing $n_{i}$ by $\frac{1}{n_{i}}$ in Eq. 1 with $i=x, y, z$.

In this work, the theoretical angle between the principal axis corresponding to $n_{z}$ and the optic axis given by Eq. 1 is used to compare the calculated wavelength dependence of $V$ with the experimental results of the optic axis direction shift at room temperature. The three principal refractive indices of KGW can be found in the literature in papers by Pujol et al. [26] for Erdoped KGW and by Konstantinova et al. [28] for Nd-doped KGW. Their values are depicted in Fig. 2. The refractive indices from Pujol et al. [26] are given with an accuracy of $10^{-3}$ from 400 to $1,600 \mathrm{~nm}$ and those from Konstantinova et al. [28] with an accuracy of $5 \times 10^{-4}$ from 430 to $640 \mathrm{~nm}$. As one can see on Fig. 2, the values of $n_{x}$ and $n_{y}$ are similar for both authors in their measurement ranges. Only the values for $n_{z}$ do not coincide for the two crystals, with a lower value observed for Nd-doped KGW. 


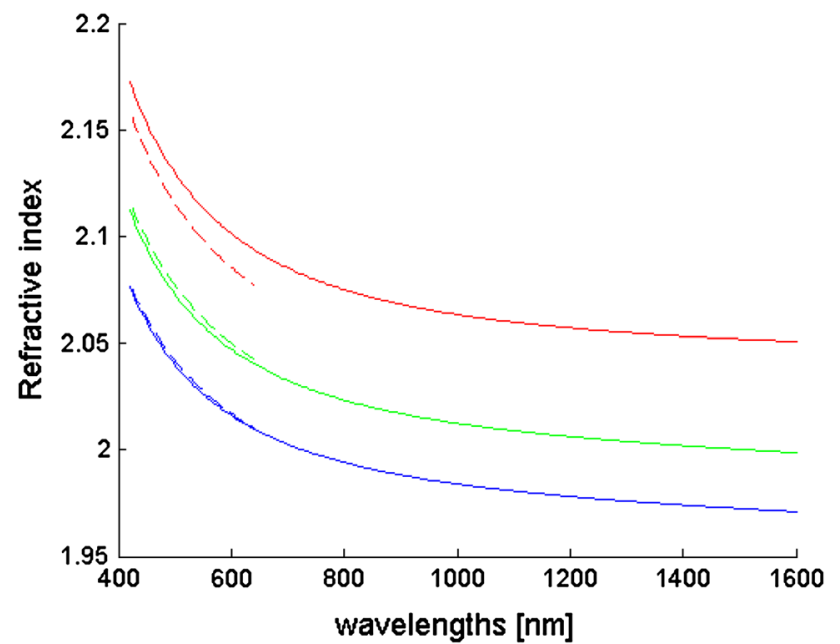

Fig. 2 Refractive index evolution of the three principal indices of $\mathrm{Er}$ doped (solid line) and $\mathrm{Nd}$ doped (dashed line) $\mathrm{KGW}$ taken from $[26,28]$

\section{Experimental setup}

The measurements of the angular variation of the optic axis orientation with wavelength were performed at LOMA in Bordeaux. The range from 430 up to $1,580 \mathrm{~nm}$ could be covered using a Ti:Sapphire laser, a compact OPO (Chameleon/Coherent) and optional frequency doubling with a BBO crystal. The wavelength of the primary Ti:Sapphire source is tunable from 680 to $1,080 \mathrm{~nm}$. With the OPO, we were able to reach the wavelength range from 1,040 up to 1,580 nm. By doubling the Ti:Sa laser, the wavelengths from 430 to $530 \mathrm{~nm}$, and by frequency doubling of the output beam after the OPO, $520670 \mathrm{~nm}$ were obtained. To ensure very precise alignment of the incident beam on the crystals for all wavelengths, the beam of the selected wavelength was injected into a single-mode optic fiber. Two different fibers have been used for the visible and the infrared range, Thorlabs P1-460A-FC-2 and P1-630A-FC2 , respectively. A collimated output $\mathrm{FC}$ connector was used to ensure that the output beam will point exactly in the same direction even when the fibers are switched. A lens ( $f=125 \mathrm{~mm}$ ) was used to focus the light inside the crystals, another one $(f=125 \mathrm{~mm})$ was used to more or less collimate the beam after the crystal, and a last one of short focal length ( $f=40 \mathrm{~mm}$ ) was used to magnify the rings for better observation, and to correct the focal variation with wavelength. Figure 3 shows a schematic drawing of the experimental setup. The two Nd-doped (at 3 at.\%) $\mathrm{KGW}$ samples with dimensions $3 \times 4 \times 11 \mathrm{~mm}$ and an undoped KGW crystal with the dimensions $3 \times 3 \times 10 \mathrm{~mm}$ were used. Each of the samples was fixed on a crystal mount which was positioned on a goniometer driven by a microcontroller with an angular step of $10^{-3}$ degrees.

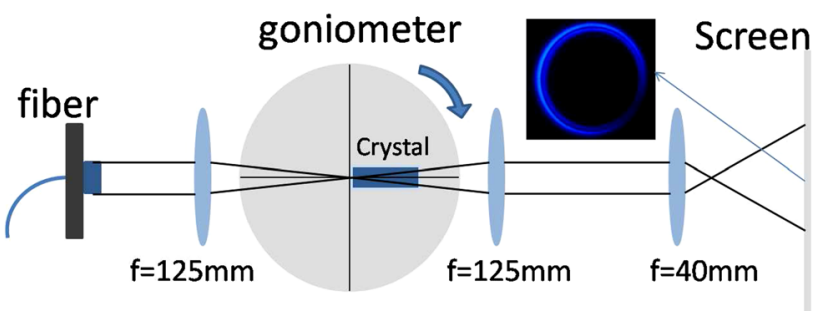

Fig. 3 Scheme of the experimental setup

The sample mount was very accurately fixed in the center of the goniometer. The doped crystals were cut for ICR at $633 \mathrm{~nm}$ with a misalignment of $1.25 \mathrm{mrad}$. Therefore, the first alignment for ICR was performed at this wavelength for one of the doped crystals by turning the goniometer until the CR rings appeared.

To ensure that the plane of variation of the optic axis was perpendicular to the rotation axis of the goniometer, we applied a simple and efficient alignment procedure. The wavelength was changed, and the goniometer was turned until the ICR was aligned in the vertical rotation axis. Instead of tilting the crystal with the horizontal rotation axis to have correct alignment, the crystal was rotated with respect to the propagation axis. To check the alignment, an upper and a lower wavelength several hundred nanometers apart were set successively and we verified that we only turn the goniometer to re-obtain the rings for both wavelengths. With this method, we ensure that the horizontal plane of the crystal is well aligned in its $X Z$ plane.

The second Nd-doped crystal was then aligned with back reflection, whereas the undoped $\mathrm{KGW}$ was the most difficult to align in the right plane due to its large cutting imprecision which leads to a deviation of $70 \mathrm{mrad}$. This difference can be explained by the fact that the crystals were bought from different companies.

As not all wavelengths were available simultaneously because of the switching from the Ti:Sapphire laser to the OPO, realignment of the fiber injection was necessary. We ensured correct reproducibility by checking the overlap for some wavelengths accessible with the different sources.

\section{Results}

The angular variation obtained with this measurement system for KGW and Nd-doped KGW is shown in Fig. 4 with a comparison to the calculated optic axis angle from the refractive indices of Er-doped $\mathrm{KGW}$ and $\mathrm{Nd}$-doped $\mathrm{KGW}$ using Eq. 1. It is important to note that the measurements performed with this method only give the relative variation of the angle. In order to compare the result with calculated values, the reference angle of $43.3^{\circ}$ and $42.1^{\circ}$ at $630 \mathrm{~nm}$ has been chosen to scale the experimental 


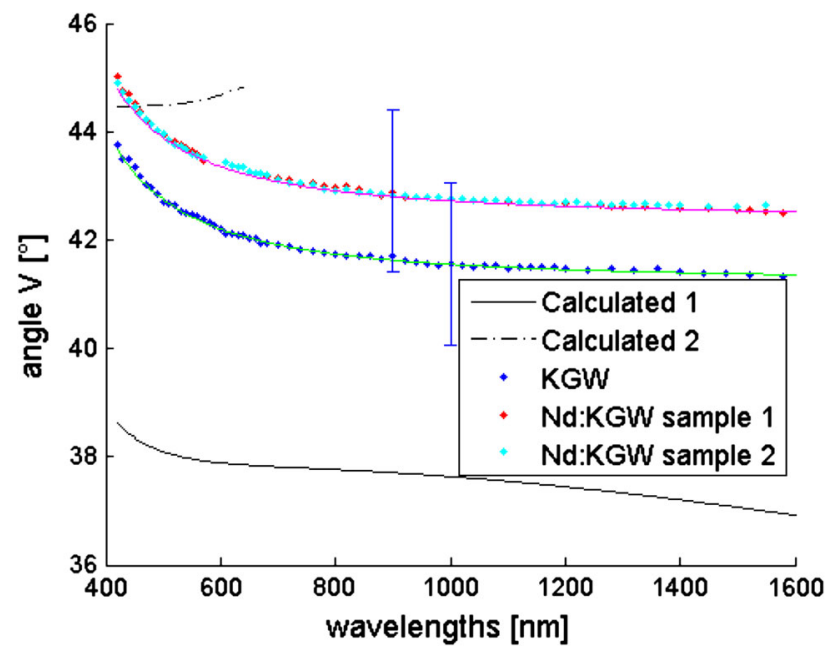

Fig. 4 Angle $V$ as a function of the wavelength (calculated values 1 and 2: obtained using Sellmeier equations from Pujol et al. [26] and from Konstantinova et al. [28], respectively, measurement of the angle $V$ variation is referenced at $43.3^{\circ}$ and $42.1^{\circ}$ at $630 \mathrm{~nm}$ for $\mathrm{Nd}$ doped $\mathrm{KGW}$ and undoped $\mathrm{KGW}$, respectively; green and pink curves are calculated using the modified $n_{z}$ refractive index)

values of Nd-doped $\mathrm{KGW}$ and undoped $\mathrm{KGW}$, respectively. Those reference angles have been determined using $\mathrm{X}$-ray measurements of the crystals. The measured variation angles of the goniometer scale have been divided by $n_{y}$, to take into account the refraction at the entrance surface.

We observe a variation of the optic axis that becomes asymptotic in the infrared region. The variation within the range of $4301,580 \mathrm{~nm}$ is about $2.4^{\circ}$. No significant difference of the curve shape is observed between the doped and undoped double tungstates. Some measurement points could not been taken for the doped KGW crystals, because of the absorption in the visible region of these samples. For example at $600 \mathrm{~nm}$, we should consider if those absorption bands could lead to a change of the birefringence and thus influence the dispersion. However, the highest variation of the birefringence due to absorption reported [28] is about $10^{-6}$ and located at $600 \mathrm{~nm}$. This is far too low to have a significant impact on the optic axis orientation and can thus be neglected.

Moreover, over the entire investigated spectral domain, we did not observe any rotation of the index ellipsoid along the crystallographic $b$-axis [corresponding to the $n_{x}$ $(X)$ direction] like it was demonstrated in other monoclinic hosts [25]. During the measurement process, only a single screw was used to align successively each wavelength for ICR. The orientation changes observed in KGW therefore can only occur in one plane within our wavelength measurement range and within the mechanical accuracy. A rotation in the other plane may exist on a very small level or for an extended measurement range.

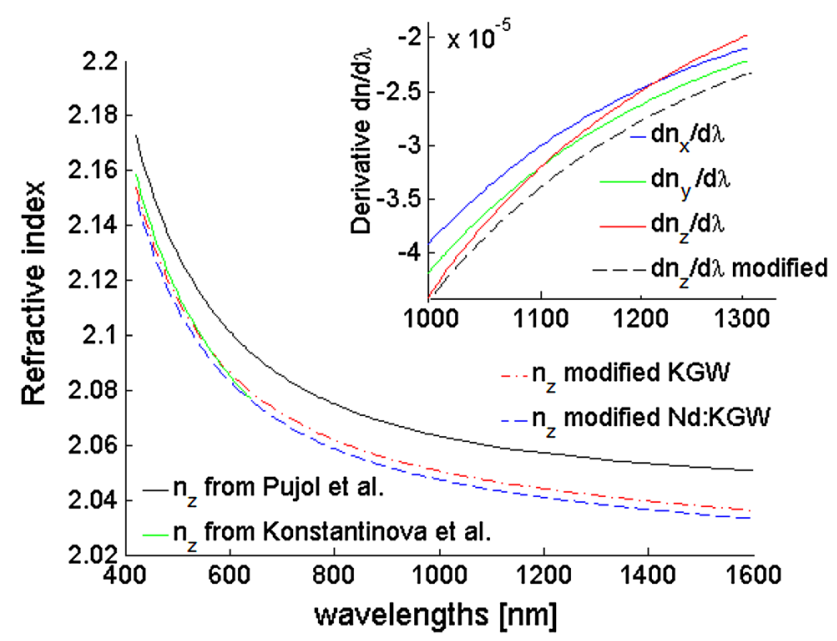

Fig. 5 Derivative of Pujol et al.'s refractive index and the modified one (inset, top right) and comparison between $n_{z}$ from Pujol et al. and the modified ones (see text)

The measurements were reproducible with an uncertainty of around $0.1^{\circ}$ in relative measurements. The uncertainty of the reference used is much higher and was determined by the X-ray diffraction measurement to approximately $1.5^{\circ}$ (indicated by the error bars in Fig. 4). For some points, the relative uncertainty is slightly higher, due to the difficulty to align for ICR with low intensity. This problem occurs beyond $1,500 \mathrm{~nm}$, and for some absorption regions of the Nd-doped samples in the visible range. However, one can observe that the shapes of the measured and the theoretical curves are significantly different in the infrared region. This arises from the extreme sensitivity of the angle $V$ on the refractive index value differences. In fact, a slight change of only one of the three refractive indices values changes the shape of the calculated graph and allows for a good agreement in the two curves. In the next paragraph, such a correction of the refraction index is shown. As can be seen in Fig. 4, the prediction calculated from the indices of reference [28] does not match our experimental result at all. This is probably due to Sellmeier fitting performed over a limited wavelength range with a limited number of data points only.

\section{Modification of the refractive index " $n_{z}$ "}

In this part, a correction will be applied to the refractive index of Pujol et al. [26] in order to bring the calculated values into agreement with the measured ones. This kind of correction cannot be applied on Konstantinova et al.'s refractive indices [28] due to the limited measurement range which does not allow us to determine an evolution of one refractive index. Using Eq. 1, it is possible to define one refractive index as a function of the angle $V$ and of the two other refractive indices. This will be used to 
Table 1 Sellmeier's coefficients of KGW's $n_{z}$ refractive index, with $C$ in $\mathrm{nm}$ and $D$ in $\times 10^{-9} \mathrm{~nm}^{-2}$

\begin{tabular}{lllll}
\hline Coefficients & $A$ & $B$ & $C$ & $D$ \\
\hline Pujol et al. & 1.3867 & 0.6573 & 170.02 & 0.2913 \\
Modified KGW & 1.535 & 0.5002 & 184.4 & 2.233 \\
Modified KGW:Nd & 1.535 & 0.4971 & 184.8 & 2.194 \\
\hline
\end{tabular}

recalculate one of the 3 refractive indices with the means of the measured angle $V$. The $n_{z}$ refractive index is the one that will be corrected for two reasons. The first one is that as shown in Fig. 2, $n_{x}$ and $n_{y}$ seem to be equal for differently doped KGW matrices. The second reason is illustrated in Fig. 5. In fact, looking at the derivatives $\frac{\mathrm{d} n}{\mathrm{~d} \lambda}$ of the indices of Pujol show that $n_{z}$ does not follow the same variation as $n_{x}$ and $n_{y}$. It is this difference that leads to the variation of the calculated angle $V$ in Fig. 4. This perturbed derivative evolution could be linked to the last measurement point of $n_{z}$ near $1,500 \mathrm{~nm}$ in Pujol et al. [26] which is not well matched by the Sellmeier fit.

In Fig. 5, the modified $n_{z}$ values with respect to the original ones from Pujol et al. are shown for comparison. The difference between Pujol et al. $n_{z}$ refractive indices and the refined ones is about 0.014 and 0.018 for undoped $\mathrm{KGW}$ and Nd-doped KGW, respectively. Therefore, a small correction of the refractive index $n_{z}$ can be derived from our experimental results. The index is given by Eq. 2 .

$n_{z}=A+\frac{B}{1-\left(\frac{C}{\lambda}\right)^{2}}-D \lambda^{2}$,

where the Sellmeier coefficients corresponding to Eq. 2 are given in Table 1.

With this new refractive index, the angle $V$ can be recalculated. Indeed, the angle $V$ calculation is very sensitive to the refractive index, for a variation of the refractive index of 0.0005 , the angle may vary by $0.15^{\circ}$. With our Sellmeier fit, it is not possible to be more precise than this value.

\section{Conclusion}

We have shown that in order to predict the evolution of the optic axis with wavelength, one must know the three refractive indices values to better than $10^{-3}$. The variation of the angle $V$ has been measured over the range from 430 to $1,580 \mathrm{~nm}$, and a correction of the $n_{z}$ refractive index taken from literature has been performed. Over the measurement range, a variation of $2.4^{\circ}$ has been measured. No significant difference of the variation has been observed between doped and undoped KGW materials. This kind of measurement can be used to check the precision of the refractive index at the $10^{-4}$ level with respect to an angular accuracy of the optic axis of $0.01^{\circ}$. Furthermore, no rotation of the index ellipsoid around the crystallographic b-axis has been observed.

\section{References}

1. H. Lloyd, On the phenomena presented by light in its passage along the axes of biaxial crystals. Phil. Mag. 1, 112 120, $207210(1833)$

2. J.C. Poggendorff, Ueber die Konische Refraction. Pogg. Ann. 124(11), 461462 (1839)

3. C.V. Raman, V.S. Rajagopalan, T.M.K. Nedungadi, Conical refraction in naphthalene crystals. Proc. Indian Acad. Sci. Sect. A, 14, 221227 (1941)

4. W. Voigt, Phys. Z. 6(6), 672673 (1905)

5. W. Voigt, Phys. Z. 6(6), 818820 (1905)

6. D.L. Portigal, E. Burstein, Internal conical refraction. J. Opt. Soc. Am. 59(12), 15671573 (1969)

7. E. Lalor, An analytical approach to the theory of internal conical refraction. J. Math. Phy. 13(4), 449454 (1972)

8. A.M. Belskii, A.P. Khapalyuk, Internal conical refraction of bounded light beams in biaxial crystals. Opt. Spectrosc. 44, 436439 (1978)

9. A.J. Schell, N. Bloembergen, Laser studies of internal conical diffraction. I. Quantitative comparison of experimental and the oretical conical intensity distribution in aragonite. J. Opt. Soc. Am. 68(8), 10931098 (1978)

10. A.J. Schell, N. Bloembergen, Laser studies of internal conical diffraction. II. Intensity patterns in an optically active crystal, $\alpha$ iodic acid. J. Opt. Soc. Am. 68(8), 10981106 (1978)

11. A.J. Schell, N. Bloembergen, Laser studies of internal conical diffraction. III. Second harmonic conical refraction in $\alpha$ iodic acid. Phys. Rev. A 18, 25922602 (1978)

12. M.V. Berry, Conical diffraction asymptotics: fine structure of Poggendorff rings and axial spike. J. Opt. A 6, 289300 (2004)

13. M.V. Berry, M.R. Jeffrey, J.G. Lunney, Conical diffraction: observations and theory. Proc. R. Soc. A 462, 16291642 (2006)

14. M.V. Berry, Conical diffraction from an $\mathrm{n}$ crystal cascade. J. Opt. A 12, 66516659 (2010)

15. V. Peet. Biaxial crystal as a versatile mode converter. J. Opt. 12, 095706 (2010)

16. V. Peet, Conical refraction and formation of multiring focal image with Laguerre Gauss light beams. Opt. Lett. 36, 29132915 (2011)

17. D.P. O'Dwyer, C.F. Phelan, Y.P. Ralovich, P.R. Eastham, J.G Lunney, J.F. Donegan, The creation and annihilation of optical vortices using cascade conical diffraction. Opt. Express 19, $25802588(2011)$

18. C.F. Phelan, J.F. Donegan, J.G. Lunney, Generation of a radially polarized light beam using internal conical diffraction. Opt. Express 19, 2179321802 (2011)

19. J. Hellström, H. Henricsson, V. Pasiskevicius, U. Bünting, D. Haussmann, Polarization tunable Yb:KGW laser based on inter nal conical refraction. Opt. Lett. 32(19), 27832785 (2007)

20. A. Abdolvand, K.G. Wilcox, T.K. Kalkandjiev, E.U. Rafailov, Conical refraction $\mathrm{Nd}: \mathrm{KGd}\left(\mathrm{WO}_{4}\right)_{2}$ laser. Opt. Express 18(3), 27532759 (2010)

21. K.G. Wilcox, A. Abdolvand, T.K. Kalkandjiev, E.U. Rafailov, Laser with simultaneous Gaussian and conical refraction outputs. Appl. Phys. B 99, 619622 (2010) 
22. J.P. Fève, B. Boulanger, G. Marnier, Experimental study of internal and external conical refractions in KTP. Opt. Commun. 105(3 4), 243252 (1994)

23. B. Boulanger, J.P. Fève, G. Marnier, G.M. Loiacono, D.N. Loi acono, C. Bonnin, SHG and internal conical refraction experi ments in $\mathrm{CsTiOAsO}_{4}$, comparison with $\mathrm{KTiOPO}_{4}$ and $\mathrm{KTiOAsO}_{4}$, for $1.32 \mathrm{mu}$;m type II SHG. Quantum Electron. IEEE J. 33(6), 945949 (1997)

24. P. Segonds, B. Boulanger, J.P. Fève, B. Ménaert, J. Zaccaro, G. Aka, D. Pelenc, Linear and nonlinear optical properties of the monoclinic $\mathrm{Ca}_{4} \mathrm{YO}\left(\mathrm{BO}_{3}\right)_{3}$ crystal. J. Opt. Soc. Am. B 21(4), 765769 (2004)

25. H. Hellwig, J. Liebertz, L. Bohaty, Linear optical properties of the monoclinic bismuth borate $\mathrm{BiB}_{3} \mathrm{O}_{6}$. J. Appl. Phys. 88(1), 240244 (2000)
26. M.C Pujol, M. Rico, C. Zaldo, R. Solé, V. Nikolov, X. Solans, M. Aguiló, F. Diaz, Crystalline structure and optical spectroscopy of $\mathrm{Er}^{3+}$ doped $\mathrm{KGd}\left(\mathrm{WO}_{4}\right)_{2}$ single crystals. Appl. Phys. B 68, 187197 (1999)

27. M. Born, E. Wolf, Principles of Optics, 7th edn. (Cambridge University Press, Cambridge, MA, 1999)

28. A.F. Konstantinova, V.V. Filippov, S.P. Palto, E.A. Ev dishchenko, K.B. Imangazieva, V.P. Orekhova, Investigation of the optical properties of $\mathrm{KGd}\left(\mathrm{WO}_{4}\right)_{2}: \mathrm{Nd}^{3+}$ crystals with allow ance for absorption. Crystallogr. Rep. 52(6), 10791087 (2007) 